\title{
EDITORIAL
}

\section{Influenza pandemic vaccines: how to ensure a low-cost, low-dose option}

\author{
Global access to an effective influenza vaccine will be essential in the event of a pandemic. \\ Here, the WHO's Klaus Stöhr, Marie-Paule Kieny and David Wood argue that assessing the \\ clinical benefits of low-dose $\mathrm{H} 5 \mathrm{~N} 1$ vaccines in animal models is crucial to achieving this goal.
}

It has been said time and again. In the event of an influenza pandemic, vaccines are the most effective means of intervention in terms of reducing cost, disease and, ultimately, death. In the same breath, it is also stressed that the supply of vaccine will be insufficient to meet demand when the pandemic virus eventually emerges and spreads around the globe. The reality is, however, for countries that do not have the capacity to produce vaccine domestically or who do not have advance purchase agreements in place, 'insufficient vaccine' could, in effect, mean 'no vaccine'.

In April 2004, WHO reference laboratories provided an $\mathrm{H} 5 \mathrm{~N} 1$ vaccine prototype influenza strain to the pharmaceutical industry. It took approximately 1 year to begin the first clinical trials using potentially pandemic viruses, including the H5N1 subtype. The shortage of vaccine production capacity and uncertainty in terms of the subtype and immunogenicity of the pandemic virus dictated the objective of these trials: finding a safe, effective and low-dose formulation so that existing production capacity could be 'stretched' to supply as many vaccine doses as possible. This 'antigen-sparing' strategy appeared very promising. An $\mathrm{H} 2 \mathrm{~N} 2$ influenza vaccine that contained less than $20 \%$ of the usual amount of antigen per dose used in a seasonal vaccine and that also used non-proprietary and cheap aluminium adjuvant produced remarkable immune responses in unprimed human volunteers. Indeed, calculations based on these findings (from 2004) suggested that it would be possible to manufacture enough $\mathrm{H} 5 \mathrm{~N} 1$ vaccine (with a two-dose formulation) for over $50 \%$ of the world's population in less than 9 months. Cautionary voices dampened this enthusiasm, however, for two main reasons: first, there are variations in efficacy between different virus subtypes in protecting against disease (the minimal amount of antigen needed per dose differs between subtypes). Second, in contrast to the interpandemic interval, the value of standard immunologic surrogates of protection in a pandemic is uncertain.

We are now realizing that these cautionary voices were correct. Results from several recently completed clinical trials using aluminium-adjuvanted $\mathrm{H} 5 \mathrm{~N} 1$ vaccines indicate that the antigen-sparing $\mathrm{H} 5 \mathrm{~N} 1$ vaccine formulations tested do not elicit the same, high antibody levels in peripheral blood as observed with seasonal vaccines. Two additional trials in humans with similar $\mathrm{H} 5 \mathrm{~N} 1$ vaccine formulations are ongoing, and these studies seem destined to confirm these results. A key question now facing decision makers is whether this matters? Will the failure of certain $\mathrm{H} 5 \mathrm{~N} 1$ vaccine formulations to elicit high antibody levels in naive individuals mean that they will not reduce severity of disease or death during a pandemic?

The answer is that it will matter if national authorities choose to recommend only those influenza $\mathrm{H} 5 \mathrm{~N} 1$ vaccine formulations that have been shown by conventional methods to comply with seasonal vaccine standards. These $\mathrm{H} 5 \mathrm{~N} 1$ vaccine formulations are likely to require either two to three times more antigen per dose compared with seasonal influenza vaccine or will contain a possibly proprietary adjuvant, and/or will be whole virion vaccines. These requirements will result in probable delays in the development of antigen-sparing pandemic vaccines, increase vaccine costs and widen the anticipated gap between supply and demand in terms of access to influenza pandemic vaccines.

\section{What can be done?}

In these circumstances of uncertainty, additional data are urgently needed to prove the clinical benefit of low-dose, aluminium-adjuvanted, split virus vaccines relative to the surrogate end-point. Although $\mathrm{H} 5 \mathrm{~N} 1 \mathrm{vac}-$ cines cannot be assessed for efficacy in humans before the pandemic, the prospects for animal trials generating information that will be useful when it comes to licensing pandemic influenza vaccines are excellent. Indeed, preliminary data obtained from studies on ferrets and non-human primates challenged with homologous and heterologous $\mathrm{H} 5 \mathrm{~N} 1$ virus indicates that aluminiumadjuvanted split virus/subunit $\mathrm{H} 5 \mathrm{~N} 1$ vaccines with lower amounts of antigen per dose provide a significant clinical benefit, including reduced disease severity and reduced viral excretion. 
Klaus Stöhr, Marie-Paule Kieny and David Wood are at the Department for Immunization, Vaccines and Biologicals, World Health Organization, Avenue Appia, CH 1211-Geneva 27, Switzerland.

e-mail: stohrk@who.int

The authors are staff members of the World Health Organization (WHO). They alone are responsible for the views expressed in this publication and they do not necessarily represent the decisions, policy or views of the WHO.
Regulatory agencies such as the US Food and Drug Administration and European Medicinal Evaluation Agency are already requesting additional studies to support decision-making on influenza pandemic vaccines. What is required now is an internationally coordinated research effort to study the efficacy or immunogenicity of selected low-dose $\mathrm{H} 5 \mathrm{~N} 1$ influenza pandemic vaccines in animal models and the linking of these findings with existing immunogenicity data from human trials. In particular, vaccines produced by the current production process (that is, split virus/subunit vaccines) using aluminium as the adjuvant should be included in these studies. This research would provide the additional evidence necessary to support the establishment of a cut-off point for haemagglutinin-inhibition seroconversion in humans and improved prediction of disease outcome. Furthermore, these studies could also help to assess the potential of aluminium-adjuvanted vaccines to cause unexpected immunopathogenic effects.

\section{How much would this cost?}

Testing a small number (8 to 12) low-dose vaccine formulations - with and without adjuvants, subunit and whole virion vaccines, and intramuscular and intradermal formulations - in ferrets and non-human primates would generate a significant amount of supporting data to inform decision-making regarding the regulation of pandemic vaccines (relating to, for example, patient survival, disease outcome and virus excretion). Costs for this are likely to be less than US\$1 million and would include the resources required for international coordination, including agreement on type of vaccines, development of research protocols, identification of partners and laboratories for animal trials and diagnosis, and evaluation of results. This amount is small, especially when it is realized that this data is essential to ensure access to low-dose vaccines enhanced with a low-cost, non-proprietary adjuvant, and manufactured without costly changes to the production process.

\section{Additional benefits}

The above-mentioned animal trials should also facilitate the success of other ongoing efforts to develop antigensparing, $\mathrm{H} 5 \mathrm{~N} 1$ vaccine formulations. They would also help in the interpretation of results from several $\mathrm{H} 5 \mathrm{~N} 1$ vaccine human trials using various adjuvants (the results of which are expected this summer). Increasingly, whole virion vaccines will be tested (to date, mostly split virus and subunit vaccines have been investigated) with the expectation that these vaccines will be more immunogenic. Currently, six clinical trials in humans are scheduled to begin this year. Tentative immunogenicity results from three trials indicate that these whole virion vaccines are more immunogenic when compared with the split virus or subunit versions. Critics are concerned, however, about the expected increase in reactogenicity (side effects). Whole virion vaccines are not, necessarily, more reactogenic than split/ subunit vaccines and improved purification will further reduce the side effects. In addition, some mild side effects might be acceptable considering the potential benefits of an influenza vaccine during a pandemic. However, as split virus and subunit vaccines constitute more than $90 \%$ of the global vaccine supply, a more pressing concern about using whole virion vaccines is that significant changes (both time- and resource-demanding) will be necessary in the vaccine manufacturing process.

Although the assertion that influenza vaccines are the most effective intervention in the event of an influenza pandemic still holds true, additional investments are urgently needed into supplementary animal trials to demonstrate the clinical benefits of low-dose $\mathrm{H} 5 \mathrm{~N} 1$ vaccines. This research can not supplant other efforts to shorten the considerable gap in access to influenza pandemic vaccines but, in the absence of this scientific data, national authorities will have little choice but to proceed with high-dose $\mathrm{H} 5 \mathrm{~N} 1$ formulations, vaccines with non-aluminium adjuvants or whole virion vaccines. This could have a major impact on the availability of an effective pandemic influenza vaccine at a time when it is needed the most. 